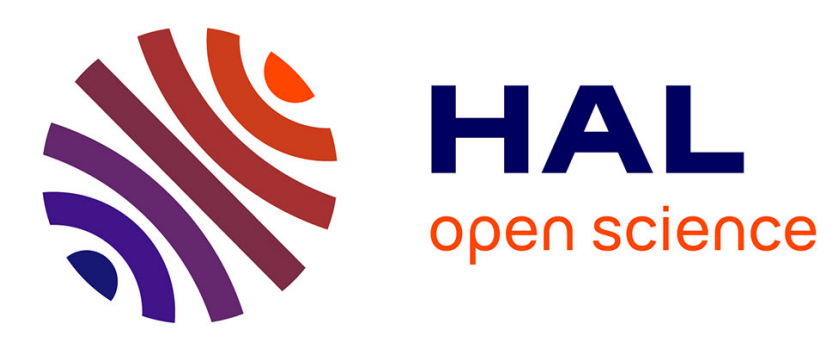

\title{
Numerical results for linear Fredholm integral equations of the first kind over surfaces in $3 \mathrm{D}$
}

\author{
Andreas Kleefeld
}

\section{To cite this version:}

Andreas Kleefeld. Numerical results for linear Fredholm integral equations of the first kind over surfaces in 3D. International Journal of Computer Mathematics, 2011, pp.1. 10.1080/00207160.2011.554543 . hal-00707582

\section{HAL Id: hal-00707582 \\ https://hal.science/hal-00707582}

Submitted on 13 Jun 2012

HAL is a multi-disciplinary open access archive for the deposit and dissemination of scientific research documents, whether they are published or not. The documents may come from teaching and research institutions in France or abroad, or from public or private research centers.
L'archive ouverte pluridisciplinaire HAL, est destinée au dépôt et à la diffusion de documents scientifiques de niveau recherche, publiés ou non, émanant des établissements d'enseignement et de recherche français ou étrangers, des laboratoires publics ou privés. 


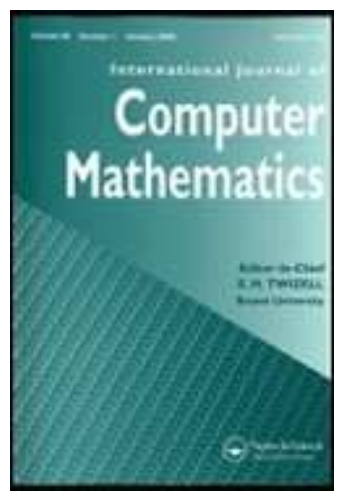

\section{Numerical results for linear Fredholm integral equations of the first kind over surfaces in 3D}

\begin{tabular}{|r|l|}
\hline Journal: & International Journal of Computer Mathematics \\
\hline Manuscript ID: & GCOM-2010-0852-B.R1 \\
\hline Manuscript Type: & Original Article \\
\hline Date Submitted by the \\
Author: & 04-Jan-2011 \\
\hline Complete List of Authors: & Kleefeld, Andreas; BTU Cottbus \\
\hline Keywords: & $\begin{array}{l}\text { Fredholm integral equations, ill-posed problems, regularization, } \\
\text { Tikhonov method, Nyström interpolation, boundary element method }\end{array}$ \\
\hline
\end{tabular}

\section{SCHOLARONE ${ }^{\text {m }}$ Manuscripts}




\title{
Numerical results for linear Fredholm integral equations of the first kind over surfaces in 3D
}

\author{
Andreas Kleefeld* \\ Brandenburgische Technische Universität Cottbus, Konrad-Wachsmann-Allee 1, 03046 \\ Cottbus, Germany \\ (Received 2 November 2010)
}

\begin{abstract}
Linear Fredholm integral equations of the first kind over surfaces are less familiar than those of the second kind, although they arise in many applications like computer tomography, heat conduction, and inverse scattering. This article emphasizes their numerical treatment, since discretization usually leads to ill-conditioned linear systems. Strictly speaking, the matrix is nearly singular and ordinary numerical methods fail. However, there exists a numerical regularization method - the Tikhonov method - to deal with this ill-conditioning and to obtain accurate numerical results.
\end{abstract}

Keywords: Fredholm integral equations; ill-posed problems; regularization; Tikhonov method; Nyström interpolation; boundary element method

AMS Subject Classification: 45B05; 65F22; 65N38

\section{Introduction}

Integral equations arise in a variety of applications ( $c f .[20])$. Depending on the field, one obtains Fredholm integro-differential equations ( $c f .[1,8]$ ), Volterra integral equations ( $c f .[17])$, or Fredholm integral equations ( $c f .[10])$. The latter case can be classified further into linear and nonlinear Fredholm integral equations of the first or second kind. Although a recent topic of research, linear Fredholm integral equations of the first kind are less familiar, since they are often ill-posed. They are usually considered only over an interval (cf. [13, 23]).

In this article, we consider Fredholm integral equations of the first kind over surfaces in three dimensions with emphasis on their numerical treatment, since they arise in various applications in physics, computer tomography, and heat conduction problems (cf. [24]).

The inverse scattering problem is still under investigation with many unanswered questions $(c f .[7,15,16])$. In the direct problem, the scattered field has to be calculated for a given obstacle and incident field. The inverse problem consists of finding the shape of an obstacle for given scattered and incident fields. This question arises in applications like sonar, radar, medical imaging, and geophysical exploration. The main issues are that the solution depends nonlinearly on the boundary and the reconstruction of the scattering wave from its far field is illposed.

There are several approaches to solve this inverse problem (cf. [22]). One method is to split the inverse scattering problem into two parts. In the linear ill-posed part,

\footnotetext{
*Email: kleefeld@tu-cottbus.de

ISSN: 0020-7160 print/ISSN 1029-0265 online

(C) 2010 Taylor \& Francis

DOI: 0020716YYxxxxxxxx

http://www.informaworld.com
} 
one must reconstruct the scattered wave from the far field pattern. The nonlinear well-posed part consists of finding the boundary of the object. More specifically, the integral equation of the first kind

$$
u^{\infty}(\hat{x})=\frac{1}{4 \pi} \int_{\Gamma} \mathrm{e}^{\mathrm{i} \kappa\langle\hat{x}, y\rangle} \varphi(y) \mathrm{d} \Gamma_{y}, \quad \hat{x} \in \mathbb{S}^{2}=\left\{\hat{x} \in \mathbb{R}^{3}:|\hat{x}|=1\right\}
$$

has to be solved for the density $\varphi$ provided the far-field pattern $u^{\infty}$ and a closed surface $\Gamma$ is given $(c f .[7])$.

In order to attack this complex inverse scattering problem for arbitrary obstacles, we first consider the ill-posed part of this problem by looking at linear Fredholm integral equations of the first kind over surfaces.

The outline of this article is as follows. Section 2 gives the preliminaries about linear Fredholm integral equations of the first kind with a smooth kernel. Singular kernels are not considered here, since they are already discussed in [3] and [5]. The next section discusses the boundary element method (BEM) for solving the first kind integral equations over a surface with emphasis on the numerical approximation. An integration scheme is presented which reduces the linear system dramatically. Another approach for more complex surfaces might be to consider meshless methods (see [21] for details and the description of the moving least squares (MLS) method). Section 4 is devoted to the Tikhonov regularization method and its usage. Furthermore, the discretization of the operator equation is given in detail. Section 5 illustrates numerical examples for the direct and inverse problem for several smooth and piecewise smooth surfaces and for different kernel and data functions. With the Nyström interpolation the solution can be obtained at every point. The last section gives a summary and an outlook for future work.

\section{Fredholm integral equation of the first kind}

In this section, the linear Fredholm integral equation of the first kind is presented.

DEFinition 2.1 (Fredholm integral equation of the first kind) We denote the equation of the form

$$
\int_{\Gamma} k(x, y) f(x) \mathrm{d} \Gamma_{x}=g(y), \quad y \in \Gamma
$$

a Fredholm integral equation of the first kind, where $\Gamma$ is a closed and bounded surface in $\mathbb{R}^{3}$. The given functions $k(x, y)$ and $g(y)$ are called the kernel of the equation and the data, respectively. The solution $f$ is an unknown function which is to be determined.

Fredholm integral equations of the first kind can be classified into two categories. The first has singular kernel functions, but those are not considered here. The second class consists of smooth functions $k(x, y)$ with non-degenerate kernel. According to Hadamard, a problem is well-posed if it has a solution (existence), not more than one solution (uniqueness), and the solution depends continuously on the data (stability). Problems are called ill-posed, if they are not well-posed. Fredholm integral equations of the first kind are examples of such ill-posed problems. That is, small changes in the data cause huge changes in the unknown function $f$ ( $c f$. [11, Section 1.1]). That means, the solution does not depend continuously on the data. Strictly speaking, the solution $f$ is extremely sensitive to small changes in $g(y)$. A method to solve an ill-posed problem approximately is called a regularization 
method. Note that the operator

$$
\mathcal{K} f=\int_{\Gamma} k(x, y) f(x) \mathrm{d} \Gamma_{x}
$$

is linear where the kernel is assumed to be smooth. For a discussion of existence and uniqueness of a solution $f$, refer to $[11,12,25]$. Note that Fredholm integral equations of the first kind over an interval are already discussed in [9, 11, 18, 24, 25].

\section{Boundary element method for solving integral equations}

The boundary element method is discussed extensively in [3] and [5] and we assume that the reader is familiar with the used notation. We briefly summarize the main points with emphasis on the numerical approximation of the Fredholm integral equation of the first kind over a surface.

\subsection{Triangulation}

Assume that $\Gamma$ is a piecewise smooth surface in $\mathbb{R}^{3}$; that means, $\Gamma$ can be written as

$$
\Gamma=\Gamma_{1} \cup \cdots \cup \Gamma_{J}
$$

Each $\Gamma_{j}$ is divided into a triangular mesh and the collection of those is denoted by

$$
\mathcal{T}_{n}=\left\{\Delta_{k} \mid 1 \leq k \leq n\right\}
$$

with mesh size

$$
\delta_{n}=\max _{1 \leq k \leq n} \operatorname{diam}\left(\Delta_{k}\right), \quad \operatorname{diam}\left(\Delta_{k}\right)=\max _{p, q \in \Delta_{k}}|p-q|
$$

We assume, that $\delta_{n} \rightarrow 0$ as $n \rightarrow \infty$. Let the unit simplex in the $s t$-plane be defined as

$$
\sigma=\{(s, t) \mid 0 \leq s, t, s+t \leq 1\}
$$

Let $\left\{q_{1}, \ldots, q_{6}\right\}$ be the three vertices and three midpoints of $\sigma$ given by

$$
\begin{aligned}
& q_{1}=(0,0), \quad q_{2}=(0,1), \quad q_{3}=(1,0) \\
& q_{4}=\left(0, \frac{1}{2}\right), \quad q_{5}=\left(\frac{1}{2}, \frac{1}{2}\right), \quad q_{6}=\left(\frac{1}{2}, 0\right) .
\end{aligned}
$$

For each $\Delta_{k}$, we assume there is a map

$$
m_{k}: \sigma \underset{\text { onto }}{\stackrel{1-1}{\longrightarrow}} \Delta_{k}
$$

which is used for interpolation and integration on $\Delta_{k}$. Define the node points of $\Delta_{k}$ by

$$
v_{k, j}=m_{k}\left(q_{j}\right), \quad j=1, \ldots, 6
$$


The first three are the vertices and the last three are the midpoints of the sides of $\Delta_{k}$. To obtain a triangulation (2) and the mapping (4), use a parametric representation for each region $\Gamma_{j}$ of (1). Assume that for each $\Gamma_{j}$, there is a map

$$
F_{j}: R_{j} \underset{\text { onto }}{\stackrel{1-1}{\longrightarrow}} \Gamma_{j}, \quad j=1, \ldots, J
$$

where $R_{j}$ is a polygonal region in the plane and $F_{j} \in C^{3}\left(R_{j}\right)$. That is, a triangulation of $R_{j}$ is mapped onto a triangulation $\Gamma_{j}$. Let $\hat{\Delta}_{k, j}$ be an element of the triangulation of $R_{j}$ with vertices $\hat{v}_{k, 1}, \hat{v}_{k, 2}$ and $\hat{v}_{k, 3}$ and mesh size $\hat{\delta}$. Then the map (4) is given by

$$
m_{k}(s, t)=F_{j}\left(u \hat{v}_{k, 1}+t \hat{v}_{k, 2}+s \hat{v}_{k, 3}\right), \quad(s, t) \in \sigma, \quad u=1-s-t .
$$

Most smooth and piecewise smooth surfaces can be decomposed as in (1). Conforming triangulations satisfying $\mathbf{T} \mathbf{1}-\mathbf{T} 3$ are considered ( $c f .[5$, p. 188]). The refinement of $\Delta_{k} \in \mathcal{T}_{n}$ is done by connecting the midpoints of the three sides of $\hat{\Delta}_{k}$ giving four new triangles. This also leads to symmetry in the triangulation and cancellation of errors occurs ( $c f .[5$, p. 173]).

\subsection{Interpolation and numerical integration}

For quadratic interpolation on $\sigma$, let $u=1-s-t$ and define the Lagrange basis functions $l_{j}(s, t), 1 \leq j \leq 6$, by

$$
\begin{aligned}
& l_{1}(s, t)=u(2 u-1), \quad l_{2}(s, t)=t(2 t-1), \quad l_{3}(s, t)=s(2 s-1) \\
& l_{4}(s, t)=4 t u, \quad l_{5}(s, t)=4 s t, \quad l_{6}(s, t)=4 s u \text {. }
\end{aligned}
$$

The corresponding basis functions $\left\{l_{j, k}(q)\right\}$ on $\Delta_{k}$ are

$$
l_{j, k}\left(m_{k}(s, t)\right)=l_{j}(s, t), \quad 1 \leq j \leq 6, \quad 1 \leq k \leq n .
$$

For a function $f \in C(\Gamma)$, define

$$
\mathcal{P}_{n} f(q)=\sum_{j=1}^{6} f\left(v_{j, k}\right) l_{j, k}(q), \quad q \in \Delta_{k}
$$

the piecewise quadratic isoparametric function interpolating $f$ on the nodes of the mesh $\left\{\Delta_{k}\right\}$ for $\Gamma$. For $f \in C^{3}\left(\Gamma_{i}\right), i=1, \ldots, J$, we have $(c f .[5$, p. 165])

$$
\left\|f-\mathcal{P}_{n} f\right\|_{\infty}=\mathrm{O}\left(\hat{\delta}_{n}^{3}\right) .
$$

To reduce integration over $\Delta_{k}$ to $\sigma$, use the map $m_{k}: \sigma \rightarrow \Delta_{k}$ to obtain

$$
\int_{\Delta_{k}} f(q) \mathrm{d} \Gamma_{q}=\int_{\sigma} f\left(m_{k}(s, t)\right) J(s, t) \mathrm{d} \sigma
$$

with the Jacobian

$$
J(s, t)=\left|\left(\frac{\partial m_{k}}{\partial s} \times \frac{\partial m_{k}}{\partial t}\right)(s, t)\right|
$$


For simplicity write the right hand side of (8) as

$$
I(f)=\int_{\sigma} f(s, t) \mathrm{d} \sigma
$$

To approximate $I(f)$, replace $f$ with the quadratic polynomial given in $(7)$ to obtain

$$
\int_{\sigma} f(s, t) \mathrm{d} \sigma \approx \sum_{i=1}^{6} w_{i} f\left(q_{i}\right), \quad w_{i}=\int_{\sigma} l_{i}(s, t) \mathrm{d} \sigma
$$

where the weights $w_{1}, w_{2}$, and $w_{3}$ are zero and $w_{4}, w_{5}$ and $w_{6}$ are $1 / 6$. The nodes $q_{i}$ are given on page (3). Thus, (10) becomes

$$
\int_{\sigma} f(s, t) \mathrm{d} \sigma \approx \frac{1}{6}\left[f\left(0, \frac{1}{2}\right)+f\left(\frac{1}{2}, \frac{1}{2}\right)+f\left(\frac{1}{2}, 0\right)\right]
$$

which has degree of precision 2.

\subsection{Approximating the surface}

For most surfaces it is difficult to find $\partial m_{k} / \partial s$ and $\partial m_{k} / \partial t$. Therefore, we approximate $m_{k}(s, t)$ in terms of $q_{1}, \ldots, q_{6}$ by

$$
\widetilde{m}_{k}(s, t)=\sum_{j=1}^{6} m_{k}\left(q_{j}\right) l_{j}(s, t), \quad(s, t) \in \sigma
$$

Thus, each $\widetilde{m}_{k}$ is a polynomial of degree $\leq 2$ in $(s, t)$ and we have $(c f .[2$, p. 33] $)$

$$
\max _{k} \max _{(s, t) \in \sigma}\left|m_{k}(s, t)-\widetilde{m}_{k}(s, t)\right|=\mathrm{O}\left(\hat{\delta}_{n}^{3}\right) .
$$

3.4 Solving integral equations of the first kind over an approximated surface Let $\left\{q_{1}, \ldots, q_{6}\right\}$ be the interpolation nodes within $\sigma$ and let

$$
v_{k, i}=\widetilde{m}_{k}\left(q_{i}\right), \quad i=1, \ldots, 6, \quad k=1, \ldots, n,
$$

which we collect in the set $\mathcal{V}_{n}=\left\{v_{1} \ldots, v_{n_{v}}\right\}$. The previous approximation technique applied to the integral equation

$$
\int_{\Gamma} k(P, Q) f(Q) \mathrm{d} \Gamma_{Q}=g(P), \quad P \in \Gamma
$$

yields the Nyström equation

$$
\sum_{j=1}^{n_{v}} w_{j} k\left(v_{i}, v_{j}\right) f\left(v_{j}\right)=g\left(v_{i}\right), \quad i=1, \ldots, n_{v}
$$


with

$$
w_{j}=\sum_{\substack{i, k \\ v_{j}=m_{k}\left(q_{i}\right)}} \widetilde{w}_{i}\left|\left(\frac{\partial \widetilde{m}_{k}}{\partial s} \times \frac{\partial \widetilde{m}_{k}}{\partial t}\right)\left(q_{i}\right)\right|, \quad \widetilde{w}_{i}=\int_{\sigma} l_{i}(s, t) \mathrm{d} \sigma .
$$

The weights $\widetilde{w}_{1}, \widetilde{w}_{2}$, and $\widetilde{w}_{3}$ are zero and $\widetilde{w}_{4}, \widetilde{w}_{5}$ and $\widetilde{w}_{6}$ are $1 / 6$. The weights $w_{j}$ are listed in [19, Example 10]. Thus, (13) is approximated by the $n_{v} \times n_{v}$ linear system

$$
\mathbf{K} f=g
$$

where $\mathbf{K}$ is the $n_{v} \times n_{v}$ matrix with the entries $w_{j} k\left(v_{i}, v_{j}\right), \boldsymbol{f}$ is an $n_{v}$-vector $\left[f\left(v_{1}\right), \ldots, f\left(v_{n_{v}}\right)\right]^{\mathrm{T}}$ and $\boldsymbol{g}$ is an $n_{v^{-v}}$ vector $\left[g\left(v_{1}\right), \ldots, g\left(v_{n_{v}}\right)\right]^{\mathrm{T}}$. Hence, the obtained linear system is of smaller size.

\section{Regularization methods}

In this chapter we introduce a well-known method which produces a stable approximation for linear ill-posed operator equations of the form

$$
\mathcal{K} x=y,
$$

where $\mathcal{K}: \mathcal{X} \rightarrow \mathcal{Y}$ is a linear and bounded operator between Hilbert spaces $\mathcal{X}$ and $\mathcal{Y}$ and $y \in R(\mathcal{X})$. The regularization method is able to calculate approximations even if the solution $x$ does not depend continuously on the data $y$, which means that the inverse of the operator is unbounded, $\left\|\mathcal{K}^{-1}\right\|=\infty$. Furthermore, the data are perturbed by noise $\delta$ which is assumed to be known, i.e.

$$
\left\|y^{\delta}-y\right\|_{\mathcal{Y}} \leq \delta
$$

The method of interest is the Tikhonov regularization. For more details on regularization theory for equations of the first kind as well as the Tikhonov method, refer to $[11,14,18,24]$.

\subsection{Tikhonov regularization}

We want to solve (15) with noisy data $y^{\delta}$. A common way to obtain a solution is to determine the best fit in the sense that we minimize the functional

$$
J(x)=\left\|\mathcal{K} x-y^{\delta}\right\|_{\mathcal{Y}}^{2}
$$

in $\mathcal{X}$. However, the solution does not depend continuously on the data. To ensure stability, we add a penalty term to the previous functional. This gives the Tikhonov functional

$$
J_{\alpha}(x)=\left\|\mathcal{K} x-y^{\delta}\right\|_{\mathcal{Y}}^{2}+\alpha\|x\|_{\mathcal{Y}}^{2} \quad \text { for } \quad x \in \mathcal{X}
$$

with regularization parameter $\alpha>0$. 
The Tikhonov functional is Fréchet differentiable for every $\alpha>0$ with the Fréchet derivative

$$
J_{\alpha}^{\prime}(x) h=2 \operatorname{Re}\left\langle\mathcal{K}^{*}\left(\mathcal{K} x-y^{\delta}\right)+\alpha x, h\right\rangle,
$$

since

$$
J_{\alpha}(x+h)-J_{\alpha}(x)-J_{\alpha}^{\prime}(x) h=\|\mathcal{K} h\|_{\mathcal{Y}}^{2}+\alpha\|h\|_{\mathcal{Y}}^{2}
$$

holds.

The Tikhonov functional $J_{\alpha}(x)$ has a unique minimum $x^{\alpha, \delta}$ in $\mathcal{X}$ for all $y^{\delta} \in \mathcal{Y}$ and $\alpha>0$. This minimum is also the unique solution of the normal equation

$$
\left(\mathcal{K}^{*} \mathcal{K}+\alpha \mathcal{I}\right) x^{\alpha, \delta}=\mathcal{K}^{*} y^{\delta}
$$

where $\mathcal{K}^{*}$ denotes the adjoint operator of $\mathcal{K}$. The operator $\mathcal{K}^{*} \mathcal{K}+\alpha \mathcal{I}$ is boundedly invertible; that means, $x^{\alpha, \delta}$ depends continuously on $y^{\delta}$.

To see this, let $x^{\alpha, \delta}$ be the minimum of the Tikhonov functional $J_{\alpha}(x)$. By the Fréchet differentiability and by the definition of a local minimum, we obtain $J^{\prime}\left(x^{\alpha, \delta}\right) h=0$ for all $h \in \mathcal{X}$. Using $h=\mathcal{K}^{*}\left(\mathcal{K} x-y^{\delta}\right)+\alpha x$ yields

$$
\left(\mathcal{K}^{*} \mathcal{K}+\alpha \mathcal{I}\right) x^{\alpha, \delta}=\mathcal{K}^{*} y^{\delta}
$$

Note that the bounded invertibility of $\mathcal{K}^{*} \mathcal{K}+\alpha \mathcal{I}$ follows from the Lax-Milgram Theorem and from the inequality

$$
\operatorname{Re}\left\langle\left(\mathcal{K}^{*} \mathcal{K}+\alpha \mathcal{I}\right) x, x\right\rangle=\|\mathcal{K} x\|_{\mathcal{Y}}^{2}+\alpha\|x\|_{\mathcal{Y}}^{2} \geq \alpha\|x\|_{\mathcal{Y}}^{2}
$$

Finally, we note that $x^{\alpha, \delta}$, defined by $x^{\alpha, \delta}=\left(\mathcal{K}^{*} \mathcal{K}+\alpha \mathcal{I}\right)^{-1}\left(\mathcal{K}^{*} y^{\delta}\right)$ minimizes $J_{\alpha}$. Obviously, the function $\lambda(t)=J_{\alpha}\left(x^{\alpha, \delta}+t h\right)$ is a polynomial of degree two with $\lambda \geq 0$ and $\lambda^{\prime}(0)=0$ for all $h \in \mathcal{X} \backslash\{0\}$, and therefore $\lambda(t) \geq \lambda(0)$ for all $t \in \mathbb{R}$ with equality only for $t=0$.

In general, the regularization parameter $\alpha$ affects two different trends. If $\alpha$ is chosen to be large, then the errors in $y^{\delta}$ will be damped. Otherwise, if $\alpha$ is chosen to be small, then $J_{\alpha}$ will be a good approximation of $J$, but the error affects the solution. To choose a good regularization parameter, we use the Morozov's discrepancy principle. It says that one should take the largest regularization parameter $\alpha=\alpha\left(\delta, y^{\delta}\right)$ such that the residual $\left\|\mathcal{K} x^{\alpha, \delta}-y^{\delta}\right\|_{\mathcal{Y}}$ is less than or equal to $\tau \delta$ for a fixed $\tau \geq 1$. That is, we find

$$
\alpha\left(\delta, y^{\delta}\right)=\sup \left\{\left\|\mathcal{K} x^{\alpha, \delta}-y^{\delta}\right\|_{\mathcal{Y}} \leq \tau \delta \quad \text { with } \quad \alpha>0\right\}
$$

where $\tau \geq 1$ is fixed. That means, we should not satisfy the operator equation more accurately than the known noise error $\delta$. It can be shown that the function $\alpha \rightarrow\left\|\mathcal{K} x^{\alpha, \delta}-y^{\delta}\right\|_{\mathcal{Y}}$ is monotonously increasing, thus we only need to find $\alpha$ such that

$$
\tau_{1} \delta \leq\left\|\mathcal{K} x^{\alpha, \delta}-y^{\delta}\right\|_{\mathcal{Y}} \leq \tau_{2} \delta \quad \text { for } \quad 1 \leq \tau_{1}<\tau_{2}
$$

holds. We can use the bisection method to find such $\alpha$. 


\subsection{Discretization of the integral operators $\mathcal{K}, \mathcal{K}^{*}$ and $\mathcal{K}^{*} \mathcal{K}$}

In order to use the previously described regularization methods, we have to discretize the operator $\mathcal{K}$ and its adjoint $\mathcal{K}^{*}$ (cf. [19, Section 5.4]). We consider the integral operator $\mathcal{K}: L^{2}(\Gamma) \rightarrow L^{2}(\Gamma)$ given by

$$
\mathcal{K} f(P)=\int_{\Gamma} k(P, Q) f(Q) \mathrm{d} \Gamma_{Q}, \quad P \in \Gamma
$$

with kernel $k \in L^{2}(\Gamma \times \Gamma)$ and $f \in L^{2}(\Gamma)$.

Discretizing (16) yields

$$
\mathcal{K} f(P) \approx \sum_{j=1}^{n_{v}} w_{j} k\left(P, v_{j}\right) f\left(v_{j}\right)
$$

with weights $w_{j}$ given in (14) and node points $\mathcal{V}_{n}=\left\{v_{1} \ldots, v_{n_{v}}\right\}$. Using the same nodes, one obtains

$$
\mathcal{K} f\left(v_{i}\right) \approx \sum_{j=1}^{n_{v}} w_{j} k\left(v_{i}, v_{j}\right) f\left(v_{j}\right), \quad i=1, \ldots, n_{v} .
$$

Thus, we obtain the $n_{v} \times n_{v}$ matrix $\mathbf{K}$ with entries $w_{j} k\left(v_{i}, v_{j}\right)$.

Next, we discretize the adjoint operator of $\mathcal{K}$. For the surface $\Gamma$ the integral operator $\mathcal{K}^{*}: L^{2}(\Gamma) \rightarrow L^{2}(\Gamma)$ is given by

$$
\mathcal{K}^{*} g(Q)=\int_{\Gamma} \overline{k(P, Q)} g(P) d \Gamma_{P}, \quad Q \in \Gamma
$$

with kernel $k \in L^{2}(\Gamma \times \Gamma)$ and $g \in L^{2}(\Gamma)$.

Discretizing (18) yields

$$
\mathcal{K}^{*} g(Q) \approx \sum_{j=1}^{n_{v}} w_{j} \overline{k\left(v_{j}, Q\right)} g\left(v_{j}\right)
$$

with weights $w_{j}$ and node points $\mathcal{V}_{n}=\left\{v_{1} \ldots, v_{n_{v}}\right\}$. Using the same nodes, one obtains

$$
\mathcal{K}^{*} g\left(v_{i}\right) \approx \sum_{j=1}^{n_{v}} w_{j} \overline{k\left(v_{j}, v_{i}\right)} g\left(v_{j}\right), \quad i=1, \ldots, n_{v}
$$

Thus, we obtain the $n_{v} \times n_{v}$ matrix $\mathbf{K}^{*}$ with entries $w_{j} \overline{k\left(v_{j}, v_{i}\right)}$.

Finally, we calculate $\mathcal{K}^{*}[\mathcal{K} f(P)](Q)$, since we also need it for the regularization 
methods. The discretization of $\mathcal{K}^{*} h(Q)$ with $h(Q)=\mathcal{K} f(P)$ results in

$$
\begin{aligned}
\mathcal{K}^{*} h(Q) & \approx \sum_{m=1}^{n_{v}} w_{m} \overline{k\left(v_{m}, Q\right)} h\left(v_{m}\right) \\
\underset{(17)}{\approx} & \sum_{m=1}^{n_{v}} w_{m} \overline{k\left(v_{m}, Q\right)} \sum_{j=1}^{n_{v}} w_{j} k\left(v_{m}, v_{j}\right) f\left(v_{j}\right) \\
= & \sum_{j=1}^{n_{v}} \sum_{m=1}^{n_{v}} w_{m} \overline{k\left(v_{m}, Q\right)} w_{j} k\left(v_{m}, v_{j}\right) f\left(v_{j}\right) .
\end{aligned}
$$

Using the same nodes, one obtains

$$
\sum_{j=1}^{n_{v}} \sum_{m=1}^{n_{v}} w_{m} \overline{k\left(v_{m}, v_{i}\right)} w_{j} k\left(v_{m}, v_{j}\right) f\left(v_{j}\right), \quad i=1, \ldots, n_{v} .
$$

We get the $n_{v} \times n_{v}$ matrix $\mathbf{K}^{*} \mathbf{K}$ with entries

$$
\sum_{m=1}^{n_{v}} w_{m} \overline{k\left(v_{m}, v_{i}\right)} w_{j} k\left(v_{m}, v_{j}\right)
$$

Note that we obtain the same entries when we multiply $\mathbf{K}^{*}$ and $\mathbf{K}$ obtained from above. To calculate the entries for $\mathbf{K}^{*} \mathbf{K}$ at the position $(i, j)$, we have to multiply the $i^{\text {th }}$ row of the matrix $\mathbf{K}^{*}$ with the $j^{\text {th }}$ column of $\mathbf{K}$. Thus, each entry of $\mathbf{K}^{*} \mathbf{K}$ is given by

$$
\sum_{m=1}^{n_{v}} w_{m} \overline{k\left(v_{m}, v_{i}\right)} w_{j} k\left(v_{m}, v_{j}\right)
$$

\section{Numerical results}

In this section, numerical results for several linear Fredholm integral equations of the first kind over different surfaces are presented. The considered integral equation is

$$
\int_{\Gamma} k(P, Q) g(Q) \mathrm{d} \Gamma_{Q}=f(P), \quad P \in \Gamma
$$

\subsection{Notations}

The components of the points $P$ and $Q$ on a surface are given by $P=\left[p_{x}, p_{y}, p_{z}\right]^{\mathrm{T}}$ and $Q=\left[q_{x}, q_{y}, q_{z}\right]^{\mathrm{T}}$, respectively. The errors are measured in the discrete $\ell^{2}$-norm

$$
\|x\|_{\ell^{2}}=\sqrt{\frac{1}{N} \sum_{i=1}^{N}\left|x_{i}\right|^{2}}
$$

where $N$ denotes the number of midpoints of the triangulation. Furthermore, we denote the normal distributed random error with mean $\mu=0$ and standard devi- 
Table 1. Absolute maximum error between the calculated solution $g^{N}$ and the exact solution $g$ and estimated order of convergence.

\begin{tabular}{ccccccc}
\hline $\mathrm{N}$ & \multicolumn{2}{c}{ Example 1} & \multicolumn{2}{c}{ Example 2 } & \multicolumn{2}{c}{ Example 3 } \\
\hline 12 & $4.9224 \cdot 10^{-1}$ & 2.8 & $9.6014 \cdot 10^{-1}$ & & $1.1394 \cdot 10^{-1}$ & \\
48 & $6.9362 \cdot 10^{-2}$ & 3.6 & $1.3301 \cdot 10^{-1}$ & 2.8 & $1.6225 \cdot 10^{-2}$ & 2.8 \\
192 & $5.6772 \cdot 10^{-3}$ & 3.9 & $1.1427 \cdot 10^{-2}$ & 3.5 & $1.2309 \cdot 10^{-3}$ & 3.7 \\
768 & $3.8181 \cdot 10^{-4}$ & 4.0 & $7.7630 \cdot 10^{-4}$ & 3.9 & $8.1183 \cdot 10^{-5}$ & 3.9 \\
3072 & $2.4314 \cdot 10^{-5}$ & & $4.9547 \cdot 10^{-5}$ & 4.0 & $5.1457 \cdot 10^{-6}$ & 4.0 \\
\hline
\end{tabular}

ation $\sigma$ on the right hand side function $f(P), P \in \Gamma$ with $\delta$. We assume that

$$
\left\|f^{\delta}-f\right\|_{\ell^{2}} \leq \delta
$$

where $\delta$ is a known noise level. We denote the error between the true solution $g_{T}$ and the calculated solution $g_{C}$ by $\epsilon$ and the error of the defect with $\hat{\epsilon}$. The parameters of the stopping rule of the Tikhonov method are $\tau_{1}=1, \tau_{2}=1.1$ and the interval for $\alpha$ is $\left[10^{-8}, 1\right]$. The number of bisection steps is denoted by $B$. The discretization of the operators is performed as explained in Section 4.2, and the colored surface represents the solution over the surface, since we cannot plot in 4D.

\subsection{The direct problem}

In this section, the direct problem is presented. We calculate the right hand side function provided the kernel function and the true solution are known. This will show that the integration over a surface works well as shown in Table 1. Note that we observe superconvergence at the collocation nodes. Define the estimated order of convergence (EOC) by

$$
\mathrm{EOC}=\log _{2}\left(\mathcal{E}_{N} / \mathcal{E}_{4 N}\right)
$$

where

$$
\mathcal{E}_{N}=\left\|g-g^{N}\right\|_{\infty}
$$

denotes the maximum error between the calculated solution $g^{N}$ and the exact solution $g$. The EOC seems to converge to four as $n$ is getting large; that is, we obtain a rate of $\mathrm{O}\left(\hat{\delta}_{n}^{4}\right)$ for smooth surfaces. This result has already been stated in [6].

\subsubsection{Example I}

The kernel function $K(P, Q)$ is 1 and $\rho(Q)=q_{x}^{2}$. The surface is a sphere with radius 1 . It is easy to check with MAPLE ${ }^{\complement}$ that the right hand side function $f(P)$ is $4 \pi / 3$.

\subsubsection{Example II}

The kernel function $K(P, Q)$ is given by $\frac{\partial}{\partial n_{Q}}|P-Q|^{2}$ and $\rho(Q)=1$. The surface is an ellipsoid with $a=1, b=0.75$ and $c=0.5$. The function $f(P)$ is given by $8 \pi a b c$.

\subsubsection{Example III}

The kernel function $K(P, Q)$ and the surface are the same as in Example II. The function $\rho(Q)$ is $q_{z}^{2}$. The function $f(P)$ is $8 \pi a b c^{3} / 3$. 
Table 2. The considered examples with different surfaces $\Gamma$, kernels $k(P, Q)$, data $f(P)$ and solutions $g(Q)$.

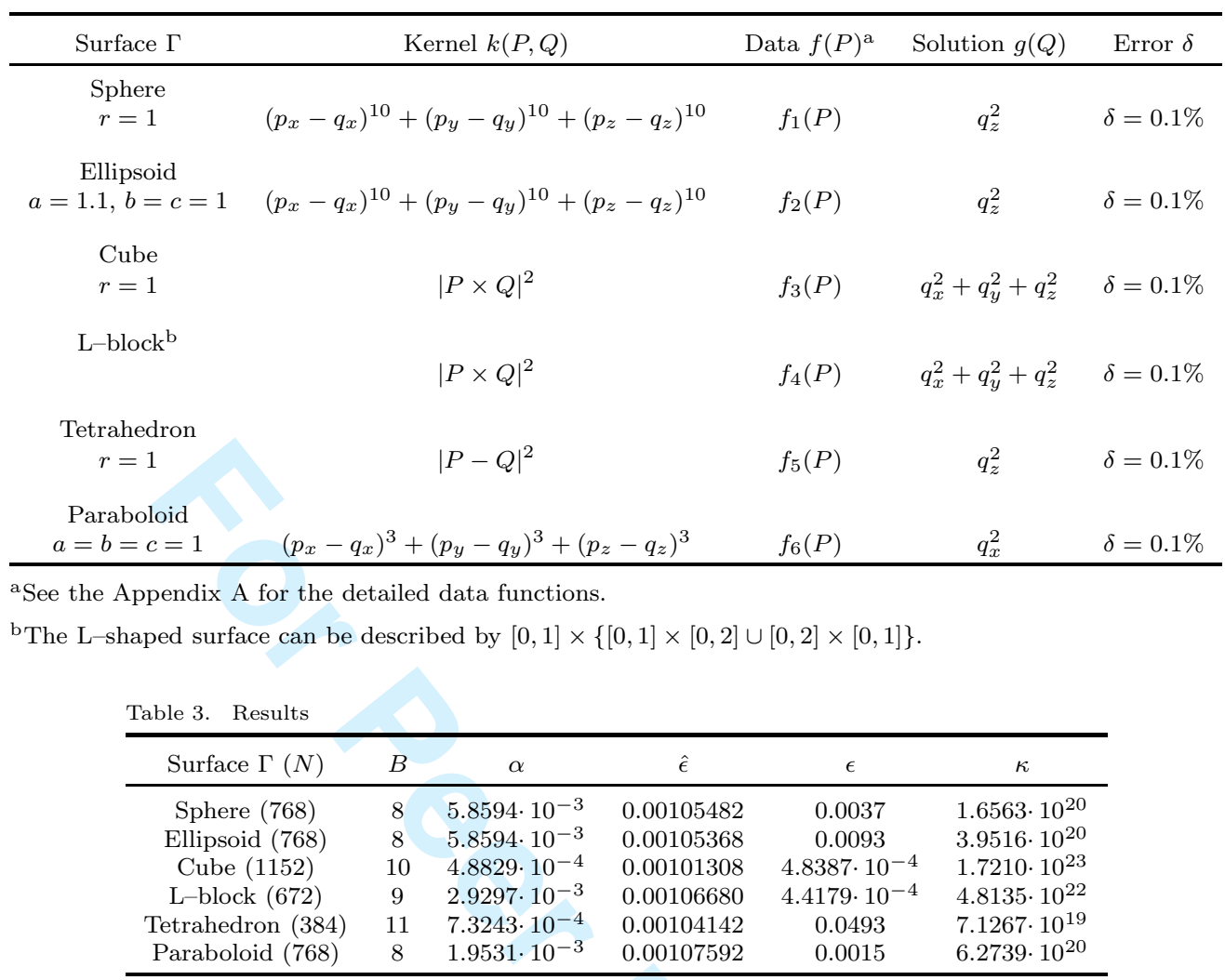

\subsection{The inverse problem}

In this section, the inverse problem is considered. We will see that we can obtain a stable approximation of the function $\rho(Q)$ for different surfaces, kernels, and right hand sides with the Tikhonov method.

Next, we consider a spherical, ellipsoidal, cubical, L-shaped, tetrahedral, and elliptic paraboloidal surface. For the setup for each example refer to Table 2. There one finds the description of the surface, the used kernel, data and solution functions, and the added error. The details of the data functions are listed in Appendix A to not impede the reading flow.

The numerical results for the six different surfaces are listed in Table 3. In this table the number of midpoints of the surface, the number of bisection steps, the regularization parameter, the error of the defect and the absolute error between the calculated and true solution are listed. In addition, the condition number of the discretized kernel matrix is presented. For more surfaces refer to [19].

As one can see in Table 3 the numerical results are very accurate, although the linear system is severely ill-conditioned. For a graphical presentation of the calculated solutions compared to the true solutions refer to the Figures 1, 2, 3, 4, 5 , and 6 , respectively.

\section{$5.4 \quad$ Nyström interpolation}

Note that by using Tikhonov regularization we obtain an integral equation of the second kind

$$
\left(\mathcal{K}^{*} \mathcal{K}+\alpha \mathcal{I}\right) \rho=\mathcal{K}^{*} f
$$




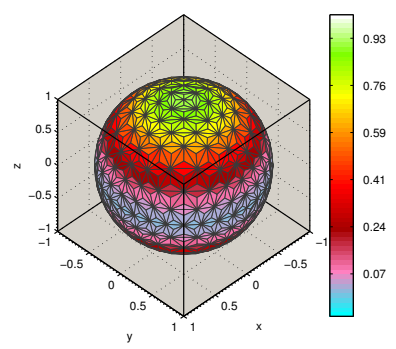

(a) True solution $q_{z}^{2}$ which is to be determined.

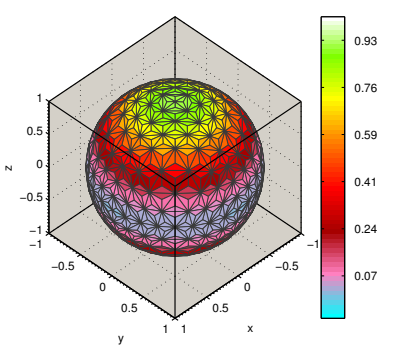

(b) Calculated solution with the Tikhonov method, $\alpha=5.8594 \cdot 10^{-3}$, and error $\delta=0.1 \%$.

Figure 1. An ill-posed inverse problem over a spherical surface.

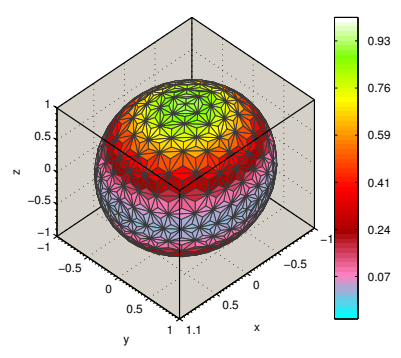

(a) True solution $q_{z}^{2}$ which is to be determined.

Figure 2. An ill-posed inverse problem over an ellipsoidal surface.

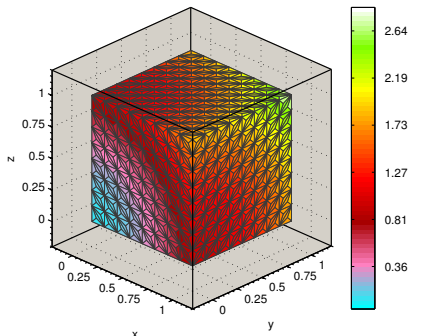

(a) True solution $q_{x}^{2}+q_{y}^{2}+q_{z}^{2}$ which is to be determined.

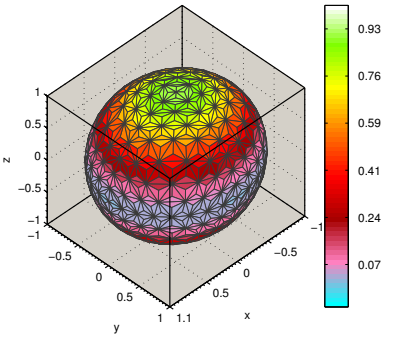

(b) Calculated solution with the Tikhonov method, $\alpha=5.8594 \cdot 10^{-3}$, and error $\delta=0.1 \%$.

Figure 3. An ill-posed inverse problem over a cubic surface.

That means, we can apply the Nyström interpolation ( $c f$. [5, Section 5.4.1]) to (19) to obtain the solution at every point $P \in \Gamma$. Discretizing (19) yields

$$
\begin{gathered}
\alpha \rho(P)+\sum_{j=1}^{n_{v}} w_{j} \overline{k\left(v_{j}, P\right)} \sum_{m=1}^{n_{v}} w_{m} k\left(v_{j}, v_{m}\right) \rho\left(v_{m}\right)=\sum_{j=1}^{n_{v}} w_{j} \overline{k\left(v_{j}, P\right)} f\left(v_{j}\right) \\
\Leftrightarrow \rho(P)=\frac{1}{\alpha} \sum_{j=1}^{n_{v}} w_{j} \overline{k\left(v_{j}, P\right)}\left[f\left(v_{j}\right)-\sum_{m=1}^{n_{v}} w_{m} k\left(v_{j}, v_{m}\right) \rho\left(v_{m}\right)\right]
\end{gathered}
$$


1

2

3

4

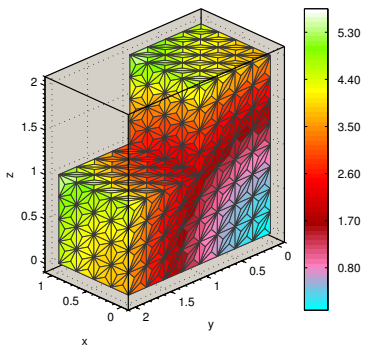

(a) True solution $q_{x}^{2}+q_{y}^{2}+q_{z}^{2}$ which is to be determined.

Figure 4. An ill-posed inverse problem over a L-shaped surface.

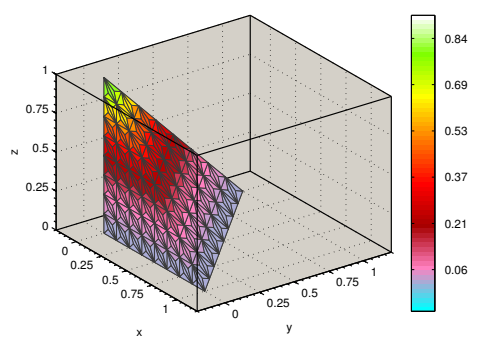

(a) True solution $q_{z}^{2}$ which is to be determined.

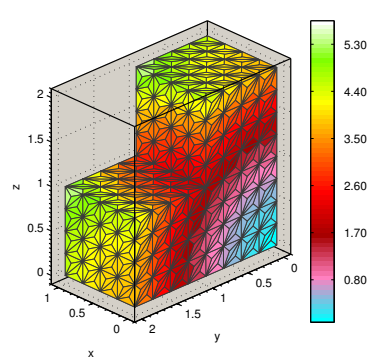

(b) Calculated solution with the Tikhonov method, $\alpha=2.9297 \cdot 10^{-3}$, and error $\delta=0.1 \%$.

Figure 5. An ill-posed inverse problem over a tetrahedral surface.

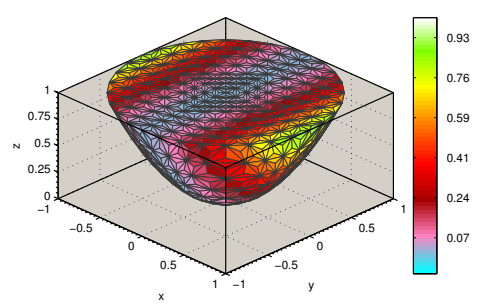

(a) True solution $q_{x}^{2}$ which is to be determined.

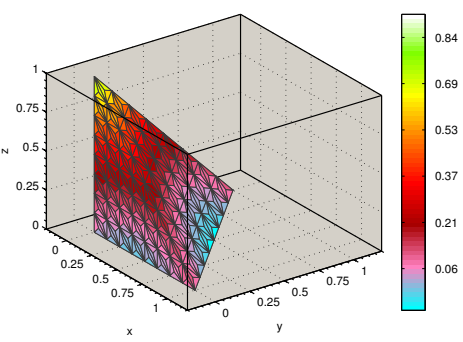

(b) Calculated solution with the Tikhonov method, $\alpha=7.3243 \cdot 10^{-4}$, and error $\delta=0.1 \%$.

\section{Figure 6. An ill-posed inverse problem over an elliptic paraboloidal surface.}

which can be solved for any point $P$ on the surface $\Gamma$ as shown in $[19$, Section 6.2.13].

When we use the spherical example, we have obtained the solution $\rho$ at 768 midpoints with $\alpha=5.8594 \cdot 10^{-3}$ and $\epsilon=0.0037$. Using the interpolation (20), we are able to calculate the solution at the remaining 258 vertices of the triangulation of the surface $\Gamma$. The maximum error between the solution at those vertices compared 
with the true solution is given by 0.00371 .

\section{Summary and outlook}

A program written in Matlab is used to approximate linear Fredholm integral equations of the first kind over surfaces with the Nyström method. It is based on a boundary element package (BIEPACK) written in Fortran which solves Fredholm integral equations of the second kind. The direct problem is solved accurately as illustrated in $\S 5.2$.

The constructed examples in $\S 5.3$ are extremely ill-posed linear integral equations of the first kind. Using standard methods, one would fail to obtain accurate numerical results. But the Tikhonov method yields accurate results, although the right hand side function is disturbed by $\delta=0.1 \%$. The regularization parameter $\alpha$ is calculated automatically by a simple bisection method which uses the interval $\left[10^{-8}, 1\right]$. Every example fulfills the stopping rule and yields accurate results. The number of iteration steps of the bisection method varies between 6 and 11. Because the Tikhonov method yields a linear integral equation of the second kind over a surface $\Gamma$, we can calculate the solution at every point $P \in \Gamma$ by the Nyström interpolation.

The numerical results of the L-shaped block and the cubical surface examples are very accurate compared to the curved surface examples, although the discretized matrix has a large condition number and we have small errors on the right hand side functions. Also, the numerical results over the curved surfaces are very accurate.

In future work we would like to apply this idea to integral equations obtained by solving the inverse scattering problems $(c f .[7,22])$.

\section{Acknowledgement}

The author thanks Professor Kendall E. Atkinson for his source code that solves the Laplace equation by a boundary element method (BIEPACK). Special thanks goes to Professor Tzu-Chu Lin for numerous fruitful discussions.

\section{References}

[1] A. Akyüz-Daşcioğlu and M. Sezer, A Taylor polynomial approach for solving high-order linear Fredholm integro-differential equations in the most general form, Int. J. Comput. Math., 84 (2007), pp. 527-539.

[2] K. E. Atkinson, Piecewise polynomial collocation for integral equations on surfaces in three dimensions, J. Integral Equations, 9 (1985), pp. 25-48.

[3] - An Empirical Study of the Numerical Solution of Integral Equations on Surfaces in $\mathbb{R}^{3}$, Reports on Computational Mathematics 1, Iowa City, November 1989.

[4] - Two-grid iteration methods for linear integral equations of the second kind on piecewise smooth surfaces in $\mathbb{R}^{3}$, SIAM J. Sci. Comput., 15 (1994), pp. 1083-1104.

[5] - The Numerical Solution of Integral Equations of the Second Kind, Cambridge University Press, Cambridge, 1997.

[6] D. Chien, Piecewise polynomial collocation for integral equations with a smooth kernel on surfaces in three dimensions, J. Integral Equations, 5 (1993), pp. 315-344.

[7] D. Colton and R. Kress, Inverse Acoustic and Electromagnetic Scattering Theory, second ed., Springer-Verlag, New York, 1998.

[8] M. Dehghan and A. Saadatmandi, Chebyshev finite difference method for Fredholm integro-differential equation, Int. J. Comput. Math., 85 (2008), pp. 123-130.

[9] L. M. Delves and J. L. Mohamed, Computational Methods for Integral Equations, Cambridge University Press, Cambridge, 1985.

[10] M. Ghasemi, E. Babolian and M. T. Kajani, Numerical solution of linear Fredholm integral equations using sine-cosine wavelets, Int. J. Comput. Math., 84 (2007), pp. 979-987.

[11] C. W. Groetsch, The Theory of Tikhonov Regularization for Fredholm Equations of the First Kind, Pitman Publishing, Marshfield, Massachusetts, 1984. 
[12] - Inverse Problems in the Mathematical Sciences, Friedr. Vieweg \& Sohn Verlagsgesellschaft, Braunschweig/Wiesbaden, Germany, 1993.

[13] C. W. Groetsch, Integral equations of the first kind, inverse problems and regularization: a crash course, J. Phys. Conf. Ser. 73, 2007.

[14] M. Hanke, Conjugate Gradient Type Methods for Ill Posed Problems, John Wiley \& Sons, Inc., New York, 1995.

[15] T. Hohage, Lecture Notes on Inverse Problems, University of Göttingen, Germany, Summer 2002.

[16] T. Hohage and H. Harbrecht, On the Numerical Solution of $3 D$ Inverse Obstacle Scattering Problems, Workshop "New Trend and Simulation and Control of PDEs", WIAS Berlin, September 2005.

[17] X. F. Li and M. Fang, Modified method for determining an approximate solution of the FredholmVolterra integral equations by Taylor's expansion, Int. J. Comput. Math., 83 (2006), pp. 637-649.

[18] A. Kirsch, An Introduction to the Mathematical Theory of Inverse Problems, Springer Verlag, New York, Inc., 1996.

[19] A. Kleefeld, Numerical results of linear Fredholm integral equations of the first kind over surfaces, Master Thesis, University of Milwaukee, Wisconsin, May 2007.

[20] A. T. Lonseth, Sources and applications of integral equations, SIAM Rev., 19 (1977), pp. $241-278$.

[21] D. Mirzaei and M. Dehghan, A meshless based method for solution of integral equations, Appl. Numer. Math., 60 (2010), pp. 245-262.

[22] R. KREss, Numerical methods in inverse obstacle scattering, ANZIAM J., 42 (2000), pp. 65-78.

[23] L. Reichel, H. Sadok and A. Shyshkov, Greedy Tikhonov regularization for large linear ill-posed problems, Int. J. Comput. Math., 84 (2007), pp. 1151-1166.

[24] A. Rieder, Keine Probleme mit inversen Problemen, Eine Einführung in Ihre stabile Lösung, Friedr. Vieweg \& Sohn Verlagsgesellschaft, Wiesbaden, 2003.

[25] G. M. Wing, A Primer on Integral Equations of the First Kind: The Problem of Deconvolution and Unfolding, SIAM, Philadelphia, Pennsylvania, 1991. 
Appendix A. The data functions

$$
\begin{aligned}
& f_{1}(P)=\frac{4}{33} r^{4} \pi\left[3 r^{10}+15 r^{8} c_{1}+110 r^{6} c_{2}+198 r^{4} c_{3}+99 r^{2} c_{4}+11 c_{5}\right] \text {, } \\
& c_{1}=p_{x}^{2}+p_{y}^{2}+9 p_{z}^{2}, \quad c_{2}=p_{x}^{4}+p_{y}^{4}+7 p_{z}^{4}, \\
& c_{3}=p_{x}^{6}+p_{y}^{6}+5 p_{z}^{6}, \quad c_{4}=p_{x}^{8}+p_{y}^{8}+3 p_{z}^{8} \text {, } \\
& c_{5}=p_{x}^{10}+p_{y}^{10}+p_{z}^{10} \text {, }
\end{aligned}
$$

where $r$ denotes the radius of the sphere.

$$
\begin{aligned}
f_{2}(P)=\frac{4}{429} a b^{3} \pi\left[c_{1}+143 c_{2}+1430 c_{3}+195 c_{4}+2574 b^{4} c_{5}+1287 c_{6}+c_{7}+c_{8}\right], & \\
c_{1}=36 b^{10}+3 a^{10}, & c_{2}=p_{x}^{10}+p_{y}^{10}+p_{z}^{10}, \\
c_{3}=a^{6} p_{x}^{4}+b^{6} p_{y}^{4}, & c_{4}=a^{8} p_{x}^{2}+b^{8} p_{y}^{2}, \\
c_{5}=p_{y}^{6}+5 p_{z}^{6}, & c_{6}=a^{2} p_{x}^{8}+b^{2} p_{y}^{8}, \\
c_{7}=2574 a^{4} p_{x}^{6}+3861 b^{2} p_{z}^{8}, & c_{8}=10010 b^{6} p_{z}^{4}+1755 b^{8} p_{z}^{2},
\end{aligned}
$$

where $a, b$ and $c$ are constants describing the ellipsoid.

$$
f_{3}(P)=\frac{1}{90} r^{6}\left(-465 p_{x} p_{y}-465 p_{x} p_{z}-465 p_{y} p_{z}+644 p_{y}^{2}+644 p_{x}^{2}+644 p_{z}^{2}\right),
$$

where $r$ denotes the side length of the cube.

$$
\begin{aligned}
f_{4}(P)= & \frac{388}{5} p_{y}^{2}+\frac{1082}{9} p_{x}^{2}-\frac{265}{6} p_{x} p_{z}+\frac{388}{5} p_{z}^{2}-\frac{389}{6} p_{y} p_{z}-\frac{265}{6} p_{x} p_{y} \\
f_{5}(P)= & \frac{1}{180} r^{4} c_{1}\left(2 r^{2}+30 c_{2}+2 \sqrt{3} r c_{3}-15 \sqrt{3} c_{4}+6 r c_{5}\right) \\
& c_{1}=7+4 \sqrt{3}, \\
& c_{3}=5-9 p_{x}-9 p_{y}+p_{z}, \quad c_{2}=p_{x}^{2}+p_{y}^{2}+p_{z}^{2} \\
& c_{5}=5 p_{x}+5 p_{y}-6 p_{z},
\end{aligned}
$$

where $r$ denotes the side length of the tetrahedron.

$$
\begin{array}{rlrl}
f_{6}(P)= & \frac{1}{110880} \pi\left(c_{1}+c_{2}+c_{3}+\sqrt{5}\left(c_{4}+c_{5}+c_{6}\right)\right), \\
& c_{1}=28644\left(p_{x}^{3}+p_{y}^{3}+p_{z}^{3}\right), & & c_{2}=41283 p_{x}+13761 p_{y}+83226 p_{z}, \\
& c_{3}=-27716-82764 p_{z}^{2}, & c_{4}=23100\left(p_{x}^{3}+p_{y}^{3}+p_{z}^{3}\right), \\
& c_{5}=12375 p_{y}+37950 p_{z}, & c_{6}=37125 p_{x}-49500 p_{z}^{2}-10300 .
\end{array}
$$

URL: http://mc.manuscriptcentral.com/gcom E-mail: ijcm@informa.com 